Bull. et Mém. de la Société d'Anthropologie de Paris, n.s., t. 10, 1998, 1-2, p. 149-167.

\title{
RISQUE ACTUEL ET RISQUE POTENTIEL DE L'ÉPIDÉMIE DE SIDA : LE CAS DES RÉGIONS FORESTIÈRES D'AFRIQUE CENTRALE [CONGO (EX ZAÏRE) - CAMEROUN]
}

\author{
Hélène Pagezy, Nicole Vernazza-Licht, Daniel Bley, \\ Ronan Boudigou, Olivier Bernard ${ }^{1}$
}

\begin{abstract}
Résumé. - L'Afrique est actuellement le continent le plus touché par le sida. Néanmoins, les rares données de séroprévalence vont dans le sens d'une Afrique centrale forestière relativement préservée de la pandémie. Ainsi, nous pensons que la forêt dans son ensemble n'est pas homogène, le risque d'exposition des populations forestieres pouvant varier sensiblement, en particulier en fonction du contexte géographique, démographique et socio-culturel. À partir d'études de cas au Congo (ex Zaïre) et au Cameroun, nous analysons différentes situations permettant d'expliquer ce décalage entre le risque actuel qui est faible et le risque potentiel lié aux déterminants de l'exposition au virus, et à ceux de la transmission.

Mots-clés : sida, risque, transmission, Afrique Centrale
\end{abstract}

PRESENT AND POTENTIAL RISKS OF THE AIDS PANDEMY: THE CASE OF THE FOREST REGIONS OF CENTRAL AFRICA (DEMOCRATIC REPUBLIC OF CONGO (EX-ZAIRE) - CAMEROON)

Summary. - Africa is presently the continent which shows the highest HIV seroprevalence. Yet, the few biological surveys available concerning the tropical forest regions are in accordance with a region preserved from the pandemy. Thus we think that the forest as a whole could present a certain heterogeneity as regards to HIV contamination, the forest people's risk of being exposed to the virus being likely to vary in relation to the geographical, demographical and socio-cultural contexts. From recent Congolese (Zairian) and Cameroonian case studies, we analyse different situations which are likely to explain the gap between the average low seroprevalence observed at present and a more important potential risk related to determinants of HIV exposition and transmission as well.

Key words: AIDS, risk, transmission, Central Africa

1. UMR 6578, Adaptabilité humaine : biologie et culture. CNRS-Université de la Méditerranée, Faculté de Médecine, Secteur Centre, 27, Bd Jean Moulin, 13385 Marseille Cedex 5. 


\section{INTRODUCTION}

En Afrique, les relations hétérosexuelles sont le premier mode de transmission du Sida chez l'adulte. Dans certains pays, il est une des premières causes de mortalité, avec les décès liés à la grossesse et à l'avortement.

La progression de l'épidémie varie à la fois selon les groupes de population et selon les régions (Verboud, 1992; Piot et al., 1992; Auvert, 1994).

Les régions forestières africaines, sont réputées comme étant faiblement touchées. Dans une étude réalisée en 1992 au Cameroun sur la région du Centre Sud, les auteurs écrivent: "Cette idée d'une certaine pureté de la brousse qui n'est pas si éloignée de l'idyllisme colonial est bien ancrée dans l'esprit des gens, non seulement des Camerounais, mais également des Européens, qu'ils soient résidents ou de passage " (Gauthier et al., 1991).

Malgré l'expansion continue du Sida sur ce continent, la forêt africaine semble ainsi un lieu préservé, vierge, à l'image même de l'immense espace forestier. Ainsi, les tests effectués dans un même village du Nord Ouest du Congo (ex Zaïre) révèlent une grande stabilité de l'épidémie à 10 ans d'intervalle, la séroprévalence s'étant maintenue à $0,8 \%$ entre 1976 et 1986 à Yambuku (Nzilambi et al., 1988). De même les données plus récentes de Froment et Mauclère (comm. pers ${ }^{(1)}$ ) au Sud Cameroun (forêt de Campo) ou en pays Tikar, comme celles de Delaporte et al. (1996) à 8 ans d'intervalle (1988-93) au Gabon (Libreville et Franceville) ne donnent pas lieu de remettre en question ces caractéristiques épidémiologiques. Les résultats de l'OCEAC de 1985-86 pour 6 états d'Afrique centrale mettent néanmoins en évidence une différence entre milieux rural et urbain (Merlin et al., 1987).

Malgré ces chiffres très bas concernant la prévalence du VIH en forêt tropicale, nous pensons que la forêt dans son ensemble n'est pas homogène, le risque d'exposition des populations forestières pouvant varier sensiblement, en particulier en fonction du contexte géographique, démographique et socio-culturel. Si le risque actuel ${ }^{(2)}$ apparait très faible au vu des résultats des enquêtes épidémiologiques, il n'est que le reflet de certaines caractéristiques des régions où ont eu lieu les prélèvements. Il s'agit de régions fortement enclavées: les voies de communication peu développées et mal entretenues (Pourtier, 1991) et les faibles densités de population (Gourou, 1953, 1960; Caldwell et Okonjo, 1969 ; Sala-Diakanda, 1980), freinent vraisemblablement la dissémination du virus. Pour

\footnotetext{
1. Prévalence Campo 0 positifs sur 292 adultes (Froment, 1984); Prévalence Yokadouma 3 positifs sur 434 adultes (Mauclère, 1995); Prévalence Bipindi 0 positifs sur 90 adultes dont 70 Pygmées (Mauclère, 1995)

2. Selon Engelhard et Seck 1989, «les tests de séropositivité peuvent être considérés comme une mesure acceptable du risque actuel, l'étude des comportements sexuels comme une appréhension correcte (du risque potentiel). Ces risques sont susceptibles de diverger considérablement en raison de la dynamique de l'épidémie, en particulier des déterminants d'une part de l'exposition au virus et d'autre part ceux de la transmission elle même. Ainsi, certains comportements sexuels pourraient donner lieu à une contagion rapide, alors que le taux actuel de séropositivité est faible, voire négligeable».
} 
notre part, nous pensons qu'il existe dans ces régions d'Afrique centrale un décalage important entre ce risque actuel qui est faible et le risque potentiel, lié aux migrations, au multipartenariat, aux maladies vénériennes non soignées, à l'existence de cas de Sida autochtones non connus de la population, et à la faiblesse de l'utilisation des préservatifs ${ }^{(3)}$.

Les résultats d'enquêtes récentes multisites par questionnaire (Caraël, 1995) ont mis en évidence que dans une population sexuellement active le multipartenariat, plutôt que la fréquence de rapports non protégés, en multipliant les situations à risque accroît celui d'être exposé au VIH (Ferry, 1996). Ainsi, l'expansion de l'épidémie dépend fortement du nombre moyen de partenaires par unité de temps. La capacité d'une personne à transmettre le virus étant plus élevée juste après son acquisition, phase de multiplication active du virus, le risque de transmission est plus élevé si cette personne a de nombreux partenaires sur une période brève plutôt que se succédant sur une plus longue durée.

Bien que les études par questionnaire aient montré que la fréquence moyenne des relations sexuelles des adultes soit faible en Afrique comme ailleurs (Caraël, 1995), les migrations, voyages, et déplacements de plus courte durée peuvent être des occasions privilégiées d'expériences sexuelles ponctuelles et occasionnelles (Pagezy, 1997).

J. Decosas (1996), qui a dressé une typologie des migrations en Afrique (migration de réfugiés, migration de réinstallation, migration internationale de la force de travail, migration intérieure et navette), rappelle à ce propos qu'il «existe une corrélation entre la mobilité et la prévalence de l'incidence de l'infection au VIH». Encore faut-il que les lieux de migration soient aussi des régions de forte prévalence au VIH et que les migrants aient des contacts non protégés avec des personnes atteintes. Du fait d'un inégal développement économique, les migrations de travail en Afrique s'effectuent surtout vers les régions littorales où le risque de contracter le VIH/Sida sont aussi plus élevés. C'est le cas des migrations entre le Burkina, le Mali ou le Niger et la Côte d'Ivoire (Painter, 1995), mais aussi entre le Sud Cameroun et le littoral gabonais.

Dans ce contexte de multipartenariat comme facteur de risque d'exposition au VIH en Afrique, Painter (1995) suggère de «prendre en compte les flux internes aux pays tout autant que les flux internationaux. Les uns comme les autres relient des zones à risque élevé à des zones à risque faible ": migrations de travail (saisonnières ou non; cf. Pison et al., 1993 ; Pison et al., 1995) ou migration de retour au village, voyages et déplacements de plus ou moins courte durée vers les villes (Boudigou et al., 1998).

L'Afrique centrale malgré son fort enclavement est une région de grande mobilité (Pagezy, 1988 ; Boudigou et al., 1998), en particulier vers des lieux moins préservés du Sida: villes et autres lieux attractifs par leur forte activité sociale et économique ${ }^{(4)}$. La vulnérabilité au VIH s'explique alors par la multiplicité et l'importance des situations temporaires de risque liées au multipartenariat pendant les déplacements. Jusqu'à présent,

3. Citons encore l'entrée précoce dans la vie sexuellement active (Aral et al., 1991 ; Caraël, 1995), et la pratique du dry sex (Brown et al., 1993).

4. Exception faite des sociétés de Pygmées, dont le rayon de mobilité est plus restreint et dont le multipartenariat s'exprime essentiellement à l'intérieur du groupe, ce qui explique en partie les résultats de Mauclère (cf. note 1 ). 
la plupart des études se sont penchées sur des groupes de personnes se trouvant dans des situations à risque "quasi permanent» : prostitué(e)s, camionneurs, migrants de longue durée, etc. Compte-tenu du contexte de la sexualité - comme le fait remarquer Ferry (1996) «il existe en Afrique un continuum d'activités extra-maritales qui entraine rétribution... Vouloir ne considérer que la prostitution "professionnelle " (commercial sex work), c'est sans doute passer à côté de la plus grande part de l'activité sexuelle et de la transmission potentielle attribuable à la prostitution" - en particulier dans les régions enclavées de forêt où nous pensons que toute personne est susceptible de se trouver de façon temporaire dans une situation à risque vis-à-vis du Sida.

C'est ce contexte de situation temporaire de risque que nous essaierons de définir à partir d'études de cas situées en milieu rural forestier, l'une dans la cuvette centrale au Congo (ex Zaïre) (sociétés Ntomba et Ekonda ${ }^{(5)}$ de la région du lac Tumba, mission ANRS, 1991), l'autre au sud Cameroun dans la région du Ntem (sociétés Ntumu et Mvae, mission UE/APFT, 1996). Indiquons que chacune de ces régions est le siège de cofacteurs favorisant la transmission du virus par voie sexuelle: l'âge précoce à la première relation sexuelle (juste après une puberté tardive, la naissance du premier enfant survient entre 16 et 18 ans), la présence de MST ulcératives ou non (gonococcie, chlamydiase, ...) mal soignées ${ }^{(6)}$; la pratique du «dry sex» (introduction de plantes asséchantes dans le vagin pour le plaisir des hommes) (Pagezy et Subervie, 1992; Pagezy, non publ.). Selon nos observations, les préservatifs sont disponibles mais peu achetés dans la région du Ntem au Cameroun et sont presque totalement absents dans la région de la Cuvette Centrale au Congo (ex Zaïre) ${ }^{(7)}$. De plus, leur qualité lubrifiée qui donne aux utilisateurs une sensation de «glissade» alors qu'ils valorisent le vagin sec (dry sex, Brown et al., 1993), est vraisemblablement un obstacle supplémentaire à leur utilisation.

5. Bien que la société Ntomba fonctionne comme une société à castes (Bahuchet, 1996), les Pygmées baTwa parlant la même langue et partageant la même culture que les cultivateurs-pêcheurs baOto mais ne s'intermarriant pas, nous ne ferons référence dans cet article qu'aux baOto qui, pour des raisons de mobilité vers des régions plus éloignées et d'échanges sexuels avec les autres groupes, sont plus vulnérables au risque de contracter le Sida.

6. Delaporte et al. (1996) rapportent qu'une étude menée à Libreville en 1994 sur les femmes enceintes, a mis en évidence la haute prévalence des maladies venériennes chez ces femmes : présence de chlamydia chez $9 \%$ d'entre elles, de gonocoques chez $2 \%$, de trichomonas chez $18 \%$ et de tréponème chez $3 \%$ d'entre elles. Ces infections seraient en partie responsables de l'infécondité en Afrique, causant l'obstruction des voies génitales supérieures: stérilité primaire et dans une moindre mesure stérilité secondaire qui résulterait d'infections ascendantes consécutives aux accouchements et avortements (Cates $e t$ al., 1985).

7. Les rapports annuels du PNLS et du Programme de Marketing Social font état d'une augmentation par région de la vente de préservatifs. Cette augmentation, si elle existe aussi en brousse, n'a vraisemblablement la même ampleur. 


\section{MÉTHODOLOGIE}

Les données originales de ce travail proviennent, pour le Congo (ex Zairre), de longs séjours répétés d'H. Pagezy dans la région du lac Tumba depuis 1970, en particulier 4 mois de terrain en liaision avec l'étude $n^{\circ} 90085$ de l'ANRS en 1991-92 (Pagezy et Subervie 1992; Pagezy, 1997). Le recueil des données a consisté en des entretiens individuels, des entretiens de groupes de 4 à 6 personnes cooptées de même sexe et d'âge voisin dans le village de Nzalekenga que nous fréquentons depuis plus de 25 ans et une constante observation par immersion dans le milieu villageois où nous séjournons (en particulier en ce qui concerne les tractations entre femmes libres et visiteurs), de longues discussions avec nos compagnons de route au cours de nos déplacements pédestres ou à vélo (suite aux conditions économiques catastrophiques, nous avons parcouru quelque $300 \mathrm{~km}$ à vélo ou à pied au cours des deux derniers séjours), avec les responsables des hôpitaux et dispensaires (médecin congolais à Bikoro, missionnaires et personnel de santé à Ntondo, médecin missionaire et autres « soeurs » à Iboko) centres de santé, ainsi qu' une expérience de vente de préservatifs discrète dans les villages que nous parcourions.

Pour le Cameroun, l'essentiel des données originales proviennent d'un questionnaire sur la fécondité des femmes $(n=325)$ et sur leurs comportements sexuels, mené pour le compte de l'Union européenne (projet APFT, 1996-98), de longues discussions avec nos proches (logeurs, informateurs, amis) et leur famille. Certaines informations proviennent de séjours plus anciens d'H. Pagezy dans la région de Kribi (étude des pêcheurs de la Lobé, 1993-95, de visite à la société forestière de Campo en compagnie de notre informateur D. Nzouango (informateur originaire de la région, travaillant dans la région avec l'équipe d'anthropologie alimentaire depuis 10 ans) ou aux plantations de palmier à huile (Socapalm) et de caoutchouc (Hévécam). Les données sur la mobilité et les migrations (Boudigou et al., 1998) ont été obtenues à partir d'entretiens semi-directifs et de longues discussions avec nos proches (informateurs, logeurs, amis) dans la région du Ntem.

\section{CONTEXTE DE LA SEXUALITÉ EN AFRIQUE CENTRALE}

Sous l'angle du multipartenariat, nous avons défini, en fonction du contexte social, économique et culturel, un certain nombre de «situations temporaires à risque : les études secondaires dans les villes, les déplacements de courte ou de moyenne durée (visites familiales, fêtes, navettes vers villes, les marchés, les sociétés forestières ou de plantation), les déplacements de plus longue durée (signifiant pour le conjoint l'abandon temporaire du domicile), l'interdit de relations sexuelles lié à l'allaitement ne touchant que les femmes, la forte mobilité conjugale, la stérilité primaire ou secondaire des femmes. 


\section{Une sexualité précoce et non réprimée}

Une entrée précoce dans la vie sexuellement active est un facteur de risque d'exposition au VIH (Caraël, 1985 ; Ferry, 1996). Au Congo (ex Zaïre) comme au Cameroun, on ne peut pas dire que l'âge au premier mariage soit très précoce (20 à 25 ans), contrairement à l'âge aux premières relations sexuelles qui ont lieu peu après une puberté tardive, soit entre 13 et 16 ans. Au Cameroun, les filles perdent leur virginité, pour la plupart à la fin de leurs études primaires (CM1 ou CM2, il ne semble pas y avoir eu de changement en une génération, les femmes plus âgées voire ménopausées rapportent la même chose lorsqu'elles ont été scolarisées); leur tout premier petit ami est en général un élève de secondaire (amour de vacances), s'il ne s'agit pas du maître (pendant l'année). Les filles qui ont fréquenté le collège (6ème ou 5ème) ont une sexualité plus tardive ; leur petit ami est encore un élève. Cette première liaison est en général éphémère. La première grossesse (pas toujours connue du géniteur) signe l'arrêt des études pour la future mère.

Au Congo (ex Zaïre) comme au Cameroun, la procréation s'inscrit dans un contexte ancien de stérilité, qui a actuellement pratiquement disparu (Sala Diakanda, 1980 ; Tabutin, 1982 ; Retel-Laurentin, 1974 ; Romaniuk, 1967). On comprend alors qu'une femme qui a mis au monde un enfant viable même hors mariage ne soit pas rejetée. Dans ce contexte un enfant, même né hors mariage, est toujours le bienvenu.

Au Congo la naissance du premier enfant est l'évènement qui donne à la femme son véritable statut dans la société, celui de mère. À cette occasion la jeune femme suit un certain nombre de prescriptions dont les effets se répercutent sur l'état de santé et psychoaffectif du couple mère-enfant (Pagezy, 1989). Une fille mère n'est pas handicapée par son statut. Seul l'enfant, qui «appartient » au clan paternel et le rejoindra tôt ou tard, souffrira le cas échéant de la séparation précoce et forcée de sa mère.

Au Cameroun comme au Congo (ex Zaïre), l'important pour une femme est d'être féconde, mais le statut d'enfant bâtard, à l'inverse du Congo, semble plus mal considéré. Si sa fille n'est pas mariée et s'il n'a pas eu lui même de garçons, le grand père maternel du bébé cherchera à en faire son fils adoptif. Il lui donnera son nom et en fera son héritier. Pour cela il ne se montrera pas pressé à recevoir la dot d'un présumé gendre peu fortuné car, tant que le père de l'enfant n'a pas versé une compensation pour celui-ci légitimant ses droits, ou n'a pas versé de dot, l'enfant n'acquiert pas la filiation de son géniteur. Si par la suite sa fille se marie, le grand père gardera cet enfant auprès de lui et ira même jusqu'à l'inscrire comme son propre fils sur son livret de famille. Il arrive aussi qu' une fille déjà mère trouve un prétendant fortuné ; elle peut lui cacher l'existence de l'enfant de peur qu'il ne rompe le mariage ou lui fasse endosser la paternité dans le cas d'une liaison précoce. L'enfant non reconnu qui vit avec ses grand-parents et les considèrent comme ses parents peut ignorer, du moins un certain temps, l'identité de sa véritable mère.

Actuellement, certaines femmes refusent, essentiellement pour des raisons psychologiques, que leur enfant soit adopté par le grand père maternel; elles acceptent leur statut de fille-mère. 
Du côté des hommes, avoir engrossé une fille sans l'avoir épousée n'est pas un déshonneur; au contraire cela peut être une raison de prestige si la jeune femme est issue d'une famille de haut statut social, directeur, ministre, ...

\section{Mariage et mobilité conjugale}

Au Congo (ex Zaïre) comme au Cameroun, la sexualité des adultes s'exerce dans un contexte normatif et moral de mariage. Néanmoins, les ruptures d'unions sont fréquentes. En principe la stérilité n'est pas une raison valable de répudier sa femme. Néanmoins, les femmes stériles sont souvent en union polygyne ; plus disponibles, elles s'adonnent souvent à des activités commerciales, voire au commerce du sexe, parfois avec la complicité du mari comme cela nous a été dit au Congo et au Cameroun ${ }^{(8)}$.

Au Congo (Pagezy, 1988), $23 \%$ des femmes de Nzalekenga, âgées de moins de 25 ans sont déjà divorcées $(n=62)$; en fin de vie génésique, $26 \%$ d'entre elles ont eu entre 4 et 6 conjoints $(n=28)$. Cette importante mobilité conjugale représente l'aspect visible du multipartenariat tel que vécu au sein des ménages car le divorce est bien souvent, au Congo comme au Cameroun, une conséquence de l'infidélité de l'un des conjoints (Pagezy, 1988).

\section{Abstinence liée à l'allaitement}

La femme qui allaite est soumise au Congo comme au Cameroun, à un interdit de relations sexuelles y compris avec son propre mari, de la fin de la grossesse jusqu'au sevrage définitif de l'enfant, soit pendant 18 à 24 mois. On pense que le sperme empoisonne le lait de la mère, mettant en danger le bébé. Si au Congo, certains couples pratiquent la «vaccination» de l'enfant (reprise progressive des relations sexuelles tout en surveillant l'état du nourrisson, voir Pagezy, 1997), au Cameroun, la plupart d'entre eux disent respecter la coutume jusqu' au sevrage ; une minorité de jeunes couples disent pratiquer le «demi-tarif» (coït interrompu) ou utiliser les préservatifs ${ }^{(9)}$ pendant cette période.

Au Congo (ex Zaïre), pour la première naissance, la période d'abstinence est encore plus longue et respectée : la jeune femme, appelée Walé, passe deux à 4 ans au sein de sa famille, surveillee de jour par un chaperon, la nuit par sa mère. Aussi longtemps qu'elle dort dans la case de «réclusion », la mère de Walé est elle aussi astreinte à l'abstinence tandis que les maris jouissent d'une certaine tolérance vis-à-vis d'éventuelles relations

8. Au Zä̈re, Soeur Marie Cécile, médecin à la mission d'lboko, seule personne en charge de cette région reculée, rapporte un cas autochtone de Sida transmis par une femme stérile vivant en polygamie, s'adonnant au commerce en général et du sexe en particulier avec la complicité de son mari. Au Cameroun, pour Céline notre informatrice, femme commerçante parcourant marchés et villages, ce sont les femmes non engagées dans une dynamique de procréation (en état de stérilité secondaire ou pratiquant la contraception), parce que plus disponibles, qui s'adonnent les relations adultérines en voyage ou même au village.

9. Comme chez les Gourou de Côte d'Ivoire (Haxaire, 1996), la période d'allaitement est pour les mêmes raisons culturelles une période pendant laquelle un couple se sent motivé à utiliser le préservatif. 
adultérines. La primipare s'empêche de commenter l'infidélité de son conjoint de peur d'être la cible de critiques l'accusant de vouloir reprendre les relations sexuelles, ce qui mettrait en danger l'enfant (Pagezy, 1997).

\section{LA GESTION DE LA SEXUALITÉ ET SES ESPACES DE TOLÉRANCE}

Dans les deux régions étudiées, les liaisons extra-conjugales des adultes se pratiquent essentiellement lors de voyages et de déplacements, c'est-à-dire hors de l'espace social du village. Lors d'un voyage, le ou la partenaire peut être occasionnel ou régulier, ce partenaire pouvant avoir de son côté un certain nombre de partenaires occasionnels ou réguliers. Le déplacement représente un espace de tolérance (Pagezy, 1997). Il libère le voyageur de ses obligations conjugales, mais aussi l'éloigne du contexte d'abstinence au sein de son ménage. Un homme de passage bien averti sera vite renseigné par la rumeur si ce n'est par la personne elle-même. Contrairement aux jeunes, les « vieux papa » disent pouvoir passer jusqu'à 2 à 3 semaines sans avoir de relations sexuelles.

\section{Les partenaires occasionnelles}

Partout en Afrique, une part des échanges entre amants consiste en cadeaux des hommes aux femmes, expression de leur affection, respect ou gratitude. «Ceux-ci ne doivent pas être considérés comme une rémunération, bien que la rumeur laisse entendre que (ces femmes) puissent se constituer ainsi un revenu confortable lorsqu'elles s'attachent deux à quatre amants généreux... Malheureusement cela est rarement le cas ... Les liaisons de courte durée ne sont qu'un moyen de survivre, non de prospérer » écrit A. Larson (1989). Il n'y a pas de prostitution au sens ou nous l'entendons (Vincke, 1991; Larson, 1989 ; Schoepf, 1988 ; Werner, 1991, Caraël, 1995b), mais un dédommagement systématique pour la femme sous forme de cadeau, de nourriture ou d'argent (Songue, 1986). Engelhard et Seck (1989) en donnent la définition suivante qui s'appliquerait assez bien ici : «Jeunes femmes célibataires ou divorcées qui acceptent fréquemment des relations sexuelles avec ou sans contrepartie monétaire de façon non protocolaire avec différents partenaires ».

Au Congo (ex Zaïr) (Pagezy et Subervie, 1992), on nomme ndumba une fille libre de tout engagement. Ce peut être une jeune célibataire de 14-16 ans, une femme divorcée ou une femme stérile (avec la complicité du mari), voire une femme temporairement libre, dont le mari est en absence de longue durée (parfois depuis plus d'une année). Ces femmes s'assument, temporairement ou non, grâce aux cadeaux ou paiement des hommes. Au village, les relations sexuelles ont lieu eu général la nuit tombée. Chaque relation amoureuse se négocie contre cadeau en nature ou en espèces. On distingue le «coup pressé » et la «nuit blanche ». En arrivant dans un village, un homme demande qu'on lui présente les femmes libres ou temporairement libres à moins qu'il ne retrouve son amie de coeur; les 
hommes adultes préfèrent les filles très jeunes ou non mariées car il craignent d'avoir des problèmes avec les femmes mariées. En effet, la prise en flagrant délit en cas de relations adultérines est très grave; elle se solde en général par une rupture d'union à charge du fautif ou du paiement d'une amende très élevée.

Au Cameroun d'une façon générale, les femmes célibataires et temporairement libres ont la liberté d'avoir des amants. Le voyage est comme au Congo (ex Zaïre) l'occasion pour un homme comme pour une femme de liaisons illicites. Ceux qui se déplacent souvent ont un amant régulier dans les lieux qu'ils fréquentent le plus (ville, marché, autre village). Lorsqu'elle voyage, son petit ami de ville entretient la femme pendant son séjour et lui laisse un somme confortable d'argent, 3 à 5000 CFA par nuit, ce qui lui permet de payer son voyage et ses habits.

Si les amants réguliers avec qui on passe la nuit sont socialement admis, les «passetemps » sont dévalorisés. Les femmes «bien» n'en ont pas ou n'en parlent pas. Avoir plusieurs partenaires par jour est mal vu. C'est le cas de femmes de la ville mais aussi de petits centres urbains. Ces femmes peuvent gagner de 3 à 4000 CFA par soirée (comme les femmes ayant un unique amant) mais avec 3 ou 4 clients ${ }^{(10)}$; elles rentrent ensuite chez leur « régulier». Ces femmes exigent que l'acte soit rapide sinon elles accusent leur partenaire de leur faire perdre leur temps et leur argent. Dans ce contexte, le rapport protégé pose problème car la durée de l'acte s'allonge et le tarif augmente.

Les femmes mariées qui ont des amants (souvent plus jeunes qu'elles) sont parmi celles qui ne sont pas ou plus engagées dans une dynamique reproductive dans laquelle périodes de grossesse et d'allaitement se succèdent (aménorrhée prolongée, stérilité secondaire, contraception volontaire). La moindre disponibilité et la fatigue sont des éléments qu'elles invoquent et qui selon elles les distinguent des femmes de la ville. Au village, la rencontre a lieu en brousse, une amie de confiance faisant le guet. En général, elles reçoivent un cadeau en nature : par exemple un pêcheur remettra un paquet de poisson même publiquement, prétextant devant le mari que le montant est déjà acquitté.

10. La journee, la passe est 500 CFA, la nuit à 1000 CFA. 


\section{LA MOBILITÉ COMME FACTEUR D'EXPOSITION AU VIH}

Au Congo (ex Zaïre) (Tableau I). comme au Cameroun les gens de la forêt sont mobiles dès leur plus jeune âge. On peut parler d'imprégnation précoce, les déplacements des enfants se faisant au sein même de l'espace social, puis dans un rayon plus important.

\begin{tabular}{|lcclllll|}
\hline & \multicolumn{4}{c}{ Hommes } & & \multicolumn{3}{c|}{ Femmes } \\
\cline { 2 - 3 } \cline { 6 - 8 } & $\mathrm{n}$ & $\%$ & remarques & & $\mathrm{n}$ & $\%$ & remarques \\
\hline $0-5$ ans & 57 & $39 \%$ & avec leur mère & & 61 & $43 \%$ & avec leur mère \\
$6-14$ ans & 86 & $35 \%$ & écoliers & & 88 & $32 \%$ & écolières \\
$15-24$ ans & 88 & $38 \%$ & & & 78 & $51 \%$ & femmes primipares \\
$25-54$ ans & 94 & $18 \%$ & & & 97 & $35 \%$ & \\
55 ans + & 25 & $12 \%$ & & & 31 & $19 \%$ & \\
\hline
\end{tabular}

Tableau I. - Pourcentage de personnes absentes plus de 3 mois de l'espace social du village (village ss et campements de pêche) de Nzalekenga au Congo (ex Zaïre) en 1979-80 en fonction du sexe et de l'âge.

\section{a) Mobilité locale, vers des zones à faible risque d'exposition}

\section{Les études primaires}

Dans les deux régions étudiées, les enfants scolarisés en primaire, pensionnaires chez un parent, passent certaines années la majeure partie de leur temps hors de leur village, pendant qu'un cousin passe l'année scolaire dans leur famille. Ils font ainsi connaissance de leur famille élargie. Les vacances scolaires sont l'occasion d'un chassé-croisé d'enfants apparentés.

\section{Les navettes et déplacements de courte durée}

Au Congo (ex Zaïre) comme au Cameroun, les déplacements de courte durée (un à trois jours) sont très fréquents. Visite familale, voyage d'affaire, deuil ou levée de deuil, fête de sortie de femme primipare Walé au Congo (ex Zaïre) (Pagezy, 1989). Citons tout particulièrement au Congo les navettes des femmes des villages voisins vers les campements de pêche (Pagezy, 1995).

\section{Le repos post-partum}

Au Congo comme au Cameroun la femme qui a accouché peut, surtout si elle est jeune, se reposer quelques mois auprès de sa famille; ce repos dure jusqu'à 4 ans au Congo dans le cas de la femme primipare Walé. 


\begin{tabular}{|c|c|c|c|c|c|c|c|c|c|c|}
\hline \multirow[b]{3}{*}{ Age } & \multicolumn{5}{|c|}{ Grande saison sèche } & \multicolumn{5}{|c|}{ Grande saison des pluies } \\
\hline & \multicolumn{2}{|c|}{ Hommes } & \multicolumn{2}{|c|}{ Femmes } & \multirow[b]{2}{*}{ Total } & \multicolumn{2}{|c|}{ Hommes } & \multicolumn{2}{|c|}{ Femmes } & \multirow[b]{2}{*}{ Tota } \\
\hline & Village & Camp & Village & Camp & & Village & Camp & Village & Camp & \\
\hline $0-3$ & 24 & 12 & 34 & 7 & 77 & 35 & 6 & 45 & 3 & 89 \\
\hline 4-12 & 66 & 11 & 56 & 9 & 142 & 66 & 2 & 67 & 5 & 140 \\
\hline $13-19$ & 36 & 17 & 27 & 4 & 84 & 50 & 8 & 48 & 3 & 109 \\
\hline $20-39$ & 17 & 48 & 41 & 22 & 128 & 51 & 27 & 55 & 10 & 143 \\
\hline $40-59$ & 27 & 20 & 40 & 5 & 92 & 36 & 11 & 36 & 5 & 88 \\
\hline $60+$ & 7 & 6 & 18 & 2 & 33 & 10 & 2 & 20 & 0 & 32 \\
\hline Total & 177 & 114 & 216 & 49 & 556 & 248 & 56 & 271 & 26 & 601 \\
\hline 16 ans et + & 67 & 87 & 118 & 30 & 302 & 124 & 45 & 156 & 16 & 341 \\
\hline
\end{tabular}

Tableau II. - Présence au village ou aux campements de pêche des hommes et femmes Ntomba de Nzalekenga (Cuvette Centrale, Congo, ex Zairre) pendant la grande saison sèche et la grande saison des pluies de 1979 (d'après Pagezy, 1988).

\section{La pêche saisonnière}

Au Congo (ex Zaïre), les pêcheurs se rendent deux fois par an en saison sèche dans les campements de pêche situés sur les grandes rivières, en juillet-aôut et janvier-février (Tableau II). Les épouses ne les y rejoignent que pendant une quinzaine de jours, une fois le travail des champs terminé, pour écoper le fretin dans la forêt inondée. De plus en plus nombreux sont ceux qui y passent la saison des pluies selon une stratégie quelque peu opportuniste, c'est-à-dire qui n'est pas identique obligatoirement d'une année à l'autre. Ce sont en particulier les maris de jeunes mères en repos postpartum (en particulier de femmes primipares Walé). Même si ce sont des déplacements que l'on peut considérer comme à faible risque épidémiologique, on doit noter qu'au niveau de ces campements de pêche, les visiteuses essaient de monnayer le poisson contre leurs charmes (Pagezy, 1995).

\section{b) Mobilité vers les villes ou zones supposées à plus grand risque}

Les villes, du fait du plus grand brassage des populations, de la présence de lieux de rencontre et d'amusement (bars, marchés), peuvent être considérées comme des endroits à plus haut risque d'exposition au Sida.

\section{Les études secondaires ou supérieures}

Les étudiants, garçons comme filles, engagés dans des études de longue durée, hébergés par la famille éloignée, pensionnaires voire locataires de meublés spécialement conçus pour eux (à Ebolowa ou Ambam au Cameroun, Mbandaka voire Kinshasa au Congo), vivent souvent en concubinage pendant cette période, même si une (ou un) fiancée les attend au village. 


\section{Les marchés régionaux et les capitales}

Au Congo (ex Zaïre), le commerce se faisait jusque vers la fin des années 80 vers la capitale régionale, Mbandaka, par camion. Hommes comme femmes empruntaient les camions «de transport " pour vendre leur manioc, régimes de plantain, gibier et poisson au marché, café, arachides et cacao à des grossistes; ils revenaient avec des articles manufacturés. Actuellement, du fait des difficultés logistiques et économiques, le marché de Mbandaka n'est plus ce qu'il était, le coût monétaire et physique du déplacement (il faut aller chercher le camion jusqu'à 30 ou $40 \mathrm{~km}$ à pied) est prohibitif; les activités commerciales sont dirigées directement vers Kinshasa par voie fluviale. Les villageois empruntent la baleinière, bateau à fond plat, pour vendre leur production de manioc, café, cacao une fois par an; le voyage durant une quinzaine de jours dans chaque sens, cela oblige les villageois à s'absenter durant au moins 3 mois. Il se peut aussi que les commerçants viennent eux-mêmes de Kinshasa en remontant le fleuve jusqu'au lac Tumba puis en empruntant les plus grandes rivières. Au cours de leur déplacement, certains d'entre eux, valise pleine de billets, proposent des relations non protégées aux femmes des villages temporairement libres et dans le besoin.

Au Cameroun, en ce qui concerne les villages de la région du Ntem, les marchés locaux de $\mathrm{Ma}$ 'an et Nyabesang permettent de faire face au plus pressé : vendre juste assez pour être dépanné en savon, sel, huile, pétrole. Le plus gros des activités commerciales se fait au marché mondial d'Abang Minko à la frontière avec le Gabon. Ce marché a été inauguré en 1990-91; il est actuellement à son apogée. Le recentrage sur Abang Minko date de 1992 avec la création du lycée d'Ambam. C'est le chef lieu de département alors qu'Ebolowa est le chef-lieu de province. Les filles «libres » sont mieux payées à Abang Minko. Elles reçoivent 4 à 5000 CFA pour la nuit. Il en coûte aussi au galant le restaurant, les boissons, les divertissements et la chambre d'hôtel, soit pas moins de 10 à $15000 \mathrm{CFA}$ la soirée. Les filles qui font à «la passe » prennent $1000 \mathrm{~F}$ à $1500 \mathrm{~F}$ par passe. Les gens viennent à Abang Minko depuis le Gabon bien qu'il faille compter une nuit et un jour de voyage depuis Libreville ${ }^{(11)}$.

\section{Les grandes sociétés forestières et les sociétés de plantation}

Au Cameroun, les grandes sociétés forestières et les grandes plantations sont des lieux de rencontre, de «fête » et de forte promiscuité les week-ends de paie. Ces sociétés emploient quelques centaines voire quelques milliers de salariés, surtout des célibataires, payés deux fois par mois : ils reçoivent une avance d'environ 1/6ème du salaire le samedi de la «quinzaine» et le solde le dernier samedi du mois. Loins de la ville, les hommes sont aussi loin des femmes. C'est pourquoi, deux fois par mois des filles viennent en car

11. Avant l'ouverture d'Abang Minko, Akong Mbang était le principal lieu d'attraction. Ce marché existe depuis 20 ans ou plus. Les Gabonais et Guinéens venaient s'y approvisionner mais il n'y avait pas de jour de "grand marché». 
depuis les grandes villes, Douala, Yaoundé, Kribi ${ }^{(12)}$; on les appelle «les arrivages». Ce sont surtout des professionnelles ${ }^{(13)}$, du moins des habituées, qui passent le week-end dans la société et repartent le lundi chez elles jusqu'au week-end suivant où elles se rendront dans une autre société pour la paie. En effet, elles «tournent » car les paies sont décalées d'une société à l' autre : d'abord les sociétés forestières de Campo et Wijma, puis la Socapalm qui exploite le palmier à huile, enfin l'Hévécam qui exploite le caoutchouc naturel. À Hévécam la fête dure plus longtemps car les salaires sont plus élevés et le grand marché qui s'y tient attire encore plus de monde ${ }^{(14)}$.

Le Dr Galega, médecin de la société Hévécam depuis presque 20 ans, a remarqué que les épisodes de gonococcies augmentaient en relation avec la paie. Comme il ne soigne gratuitement l'employé que si celui-ci se présente avec sa partenaire, il remonte la chaine de contamination. Ainsi, une « arrivage » lui a cité 32 partenaires. On retrouve pratiquement le modèle décrit par Decosas (1996) au sujet des grandes sociétés de forêt en Côte d'Ivoire.

Au Congo (ex Zaïre), suite à la nationalisation imposée, les grandes sociétés de plantation, café, cacao, palmier à huile, caoutchouc, ne sont plus des lieux d'attraction pour les populations voisines. Il n'y a plus de fête les jours de paie, car les salaires ne sont pas réevalués et arrivent très irrégulièrement (Tardieu, 1992). Les Soeurs de Bikoro qui continuent à y faire la surveillance nutritionnelle parlent même de malnutrition aigüe, voire de mouroir. Dans ce contexte, la situation est évidemment différente du Cameroun.

\section{CONCLUSION}

Si le risque actuel de contracter le Sida est faible dans la majeure partie de la forêt d'Afrique centrale (en particulier dans les zones rurales à faible prévalence), le risque potentiel est à notre avis non négligeable: risque d'exposition dans la mesure où la population, fortement mobile, se déplace vers des lieux supposés à plus forte prévalence pour le VIH (villes, sociétés de plantation, grands marchés, $c f$. Boudigou, 1998), risque de transmission compte-tenu du contexte général de la sexualité dans ces régions. La fréquence des maladies vénériennes et le contexte de stérilité, caractérisant un certain nombre de situations temporaire «à risque» (Schrijvers et al., 1991), pourraient alimenter le cas échéant la dynamique de l'épidémie. Néanmoins on ne peut pas présumer de la vitesse de propagation du virus, du moins tant qu'il n'existe pas dans la région de cas

12. Selon le PNLS, la prévalence du VIH chez les prostituées de Yaoundé était de $25 \% ; 45 \%$ chez celles de Douala.

13. Il n'y a pas que des habituées puisque quelques femmes «bien » s'y rendent en cachette arrondir leur fin de mois (observation faite par notre informateur D. Nzouango, originaire de la région et résidant à Yaoundé, à La Forestière de Campo).

14. Les week ends de paie, l'Hévécam abrite le plus grand marché de la région Sud. 
autochtones ${ }^{(15)}$, ce qui pourrait être le cas de la région du Ntem ${ }^{(16)}$, mais non de la province de l'Est ni de celle de l'Océan (Kribi) au Cameroun, ni des sociétés de plantation ${ }^{(17)}$, ni même de la Cuvette Centrale au Congo (ex Zaïre) en $1991^{(18)}$. Compte-tenu de la grande mobilité des populations, en particulier entre des régions de faible et de forte prévalence, il est vraisemblable que la dynamique de transmission du virus se fera par étapes, par exemple au niveau de centres régionaux (villes, marchés, sociétés forestières), lieux de rencontre et de brassage. Les employés célibataires des sociétés forestières ou de plantation, originaires de villages de forêt, joueraient le rôle de noyau de diffusion secondaire (core group $^{(19)}, c f$. Ferry, 1996) lors de leurs déplacements (cf. ci-dessus) et leur retour au village (Boudigou et al., 1998) (le noyau primaire étant représenté par l'ensemble des prostituées (sex workers) venues des villes à l'occasion de la paie. Le contexte du multipartenariat lié à la mobilité et aux espaces de tolérance dans la pratique quotidienne de la sexualité, favorisera ensuite sa propagation. Il semble qu'actuellement la composante économique de toute prestation sexuelle pourrait comme au Congo (ex Zaïre), être un frein aux liaisons adultérines ${ }^{(20)}$. La faiblesse du risque actuel ne sous-entend pas pour autant une stabilisation de la prévalence. Delaporte et al. (1996) suggèrent plutôt un stade épidémique précoce pouvant démarrer rapidement au cours des prochaines années. Actuellement, les chaines de contagion dans ces régions enclavées à faibles densités de population, si elles émergent

15. Au Zaïre la population dit pratiquer une stratégie de prévention en évitant d'avoir des relations sexuelles avec les étrangers ou toute personne ayant transité par une grande ville. Néanmoins, dans un contexte aigu de crise, les femmes et en particulier les femmes seules acceptent consciemment les relations non protégées avec des voyageurs et commerçants de passage. Par ailleurs, en 1991 les hôpitaux les plus proches du village d'étude font déjà etat de quelques cas de Sida autochtones. Il s'agit toujours de diagnostic clinique, les examens biologiques n'étant pas disponibles. La population ignore l'existence de ces cas et de ce fait n'est pas motivée à prendre ses dispositions, car elle ne perçoit pas la proximité de la maladie (Pagezy et Subervie, 1992).

16. L'hôpital de Ma'an ne peut pas attester de cas autochtone bien que la rumeur ait identifie $\mathbf{2}$ cas.

17. Le Dr Galega, médecin chef, fondateur de l'hôpital en 1981, indique pour la société Hévécam, sur un échantillon représentatif de 500 adultes, une prévalence de $5 \%$ (test Elisa, confirmé par WB, identique à celle des grandes villes, et plus de 5 fois supérieure aux données des Drs Froment et Mauclère, voir note 1) qui peut atteindre $12 \%$ dans certains « villages » (Galega, comm. pers.).

18. Entre 1989 et 91,18 tests positifs à l'hôpital de Bikoro (petit centre administratif au bord du lac qui draine 4 à 5000 personnes); sur 14 cas, 11 viennent du lac dont 9 de Bikoro, 1 de Mbandaka (capitale regionale), 2 cas autochtones (villages). A l'hôpital d'Iboko, village très enclavé tenu par des Soeurs missionnaires, sur 35 cas diagnostiqués, 13 viennent des lacs, 2 d'Iboko, 12 des capitales régionale (Mbandaka) et nationale (Kinshasa), 7 cas autochtones (villages). Sur 27 professions connues, 16 commerçant(e)s ou femmes ayant pratique temporairement le commerce du sexe, 5 cultivateurs, 3 enseignants, 2 pêcheurs, 1 étudiant.

19. Plummer (cite par Ferry, 1997) definit le core group comme une sous-population dont chaque individu transmet une MST à plus d'une personne susceptible. Cela recouvre un noyau de transmission; la question est de savoir si un noyau (les travailleurs des plantations) a des échanges avec d'autres sous-populations qui sont à moindre risque (les villageois), sinon l'épidémie reste cantonnée dans le noyau.

20. En 1991, les informateurs avec qui nous voyagions de village en village n'ont engagé de tractations avec les filles dans les villages que grâce à l'avance sur salaire que nous leur faisions. Actuellement seuls les enseignants, les pêcheurs, les fonctionnaires et les commerçants, qui jouissent d'un meilleur pouvoir économique ou d'un grand prestige, ont plus facilement accès aux femmes. 
simultanément à plusieurs endroits, n'atteignent pas un niveau suffisant permettant de passer au stade épidémique. Compte tenu de la présence importante dans ces régions d'infections des voies génitales, facteurs augmentant le risque de transmission du virus, on pourrait s'attendre au moins dans les villes à des fortes prévalences du VIH proches des valeurs observées dans les grandes villles d'Afrique de l'Ouest ou de l'Est. Les faibles prévalences du VIH observées en Afrique centrale obligent à considérer l'action d'autres facteurs tels que la grande diversité des sérotypes du virus ou un contexte particulier de la dynamique de transmission. Nous suggérons que la surveillance épidémiologique du monde rural en Afrique centrale doive passer par l'étude de sites intermédiaires comme les villes secondaires, les grands marchés et les grandes sociétés d'exploitation hébergeant de nombreux employés célibataires, lieux de migration et de contact entre le monde rural et le monde urbain.

Les études qualitatives n'ont pas, on le sait pas, la prétention de conduire à des conclusions généralisables mais plutôt d'apporter les éléments de réflexion préalables à des enquêtes quantitatives de type épidémiologique.

\section{Remerciements}

Nous remercions l'Agence Nationale de Recherche sur le Sida (contrat 90 085) et la Communauté européenne (projet Avenir des Peuples des Forêts Tropicales) pour leur aide matérielle. Nous remercions également les Dr Delaporte, Froment et Mauclère qui ont bien voulu nous communiquer leurs données sérologiques sur le VIH concernant l'Afrique centrale forestière. 


\section{BIBLIOGRAPHIE}

Aral (S.), Fullilova (R.), Coutinho (R.), Van Den Hoeck, 1991. Demographic and societal factors influencing risk behaviors. In : Weisserheit, J., Aral, S, Holmes, K. (Eds). Research issues in human behavior and sexuelly transmitted diseases in the AIDS area. American Society for Microbiology, Washington DC, pp. 161-176.

Auvert (B.), 1994. Epidémiologie du Sida en Afrique In: J. Vallin (Ed). Populations africaines et sida. Collection Recherches, La Découverte/CEPED, Paris, pp. 63-117.

BAHUCHET (S.), 1996. Fragments pour une histoire de la forêt africaine et de son peuplement: les données linguistiques et culturelles. In: C.M. Hladik et al. (Eds). L'alimentation en forêt tropicale. Interactions bioculturelles et perspectives de développement. UNESCO/MAB, Paris, pp. 97-120.

Boudigou (R.), Bley (D.), Pagezy (H.), Vernazza-Licht (N.), 1998. Le retour au village des migrants comme exemple des interactions ville-forêt au sud Cameroun, In: ORSTOM/SEH, Urbanisation et environnement dans les pays en développement.

Brown (J.), Ayowa (O.), Brown (R.), 1993. Dry sex and tight : sexual practices potential AIDS risk in Zaïre. Social Science and Medicine, 37 : 989-994.

Caldwell (J.C.), Okonjo (C.), 1969. La population de l'Afrique tropicale. The Population Council, New York.

CARAÉL (M.), 1995a. Sexual behaviour. In: J. Cleland et B. Ferry (Eds). Sexual behaviour and AIDS in the Developing World. Taylor and Francis, London.

Caraêl (M.), 1995b. La mesure de l'activité sexuelle dans les pays en développement. In : N. Bajos et al. (Eds). Sexualité et Sida, recherches en sciences sociales, ANRS, collection Sciences sociales, pp. 57-80.

Cates (W.), Farley (T.), Rowe (P.), 1985. Worldwide pattern of infertility: is Africa different? Lancet, ii, 596-598.

Delaporte (E.), Janssens (W.), Peeters (M.), Buve (A.), Dibanga (G.), Perret (J.L.), Dimtsambou (V.), Mba (J.R.), Courbot (M-C.G.), Georges (A.), Bourgeois (A.), Samb (B.), Henzel (D.), HeyndrickX (L.), Fransen (K.), VAN DeR Groen (G.), LarouzE (B.), 1996. Epidemiological and molecular characteristics of HIV infection in Gabon, 1986-1994. AIDS, 10: 903-910. 
Engelhard (P.), Seck (M.), 1989. Comportements sexuels et contexte culturel. Approche méthodologique de la prévention du Sida. Plurale, 1 : 83-89.

FERRY (B.), 1996. Systèmes d'échanges sexuels et transmission du VIH/Sida dans le contexte africain, communication Colloque international Sciences sociales et sida en Afrique, bilan et perspectives, Dakar, octobre 1996, abstracts 1:113-128.

GAUTHIER (J.G.), 1991. Appréhension subjective et vécu social du SIDA dans les populations urbaines et rurales du Cameroun et du Togo. Rapport de projet ANRS, Sciences de l'Homme et de la Société appliquées au SIDA. fascicule 1 : les milieux ruraux, $80 \mathrm{p}$; fascicule 2 : les milieux urbanisés, 61 p. CNRS, GDR 892/ ORSTOM/ANRS.

Gourou (P.), 1953. Les pays tropicaux, principes d'une géographie humaine et économique. P.U.F, Paris 1-6.

Gourou (P.), 1960. Atlas général du Congo, ARSOM, Bruxelles

Haxaire (C.), 1996. Prévention du Sida et levée d'interdits sexuels en pays Gouro, conséquences sociales. Communication au colloque Sciences sociales et Sida en Afrique; bilan et perpectives. Codesria, CNLS/ORSTOM, Dakar, octobre 1996, pp. 471-480.

LARSON (A.), 1989. Social contextof Human Immunodeficiency Virus transmission in Africa: Historical and Cultural bases of East and Central African sexual relations. Review of infectious disease, 2: 716.

Merlin (M.), Dupont (A.), Josse (R.), Delaporte (E.), Kollo (B.), Durand (J.P.), Limbassa (J.), Danyod (M.), hamono (B.), Diallo (S.), Senga (J.), Owonoo (A.), Kouka-Demba (D.), GEORGES (A.J.), 1987. Infection des populations par le VIHI dans 6 états d'Afrique Centrale. Document ronéo, $8 \mathrm{p}$.

Nzilambi (N.), de Cock (K.M.), Forthal (D.N.), Francis (H.), R yder (R.W.), Malebe (I.), Getchell. (J.), LAGA (M.), PIOT (P.), MC CoRMICK (J.B.), 1988. The prevalence of infection with human immunodeficiency virus over a 10-year period in rural Zaïre. New England Journal of Medicine, 31 : 276-279.

PAGEZY (H.), 1988. Contraintes nutritionnelles en milieu forestier équatorial liées à la saisonnalité et la reproduction: réponses biologiques et stratégies de subsistance chez les ba-Oto et les ba-Twa du village de Nzalekenga (lac Tumba, Zaïre). Thèse de Doctorat d'Etat es-Sciences, Université d'Aix-Marseille III, 489 p.

PAGEZY (H.), 1989. Feeding the primiparous mother among the Ntomba of Zaire. In : C.M. Hladik, $S$. Bahuchet et $I$. de Garine (Eds). Food and nutrition in the African rain forest. UNESCO/ MAB/CNRS, Paris, pp. 89-91. 
PAgezy (H.), 1995. De l'adaptation nutritionnelle à la malnutrition: interactions écologiques et socio-culturelles dans l'alimentation des Ntomba du Zaïre; In : R. Devisch, F. de Boeck et D. Jonckers (Eds) Alimentations, traditions et développements en Afrique intertropicale. l'Harmattan, Paris, pp. 272-303.

PAgezy (H.), 1997. Contexte socio-culturel de l'épidémie dans une région forestière enclavée du Zaïre: interdits de relations sexuelles et espaces de tolérance. ANRS/ORSTOM.

PAgezy (H.), SuberVIE (A.M.), 1992. Sida et modification des comportements sexuels: le cas des reclusions de longue durée chez les Mongo du sud du Zaïre, Rapport ANRS projet $n^{\circ} 90085$, $105 \mathrm{p}$ ronéo.

PaInTER (T.), 1995. Les stratégies de vie mobiles: un défi aux programmes de lutte contre le VIH/ Sida en Afrique. Sociétés d'Afrique et Sida, 10: 5-6.

Piot (P.), LaDa (M.), Goeman (J.), 1992. Afrique : une épidémie à deux virus et à plusieurs vitesses. Le Journal du Sida, pp. 14-18.

Pison (G.), Le Guenno (B.), Lagarde (E.), Enel (C.), Seck (C.), 1993. Seasonal migration : a risk fcator for HIV infection in rural Senegal. Journal of Acquired Immune Deficiency Syndromes, $6: 196-200$.

Pison (G.), Lagarde (E.), Enel (C.), Desgrées du Lou (A.), 1995. Comportements sexuels, migrations saisonnières et risques d'infection par le VIH et les maladies sexuellement transmissibles au Sénégal, Sociétés d'Afrique et sida, $1:$ 8-10.

Pourtier (R.), 1991. L'inéluctable défi des transports, Politique africaine, $\mathrm{n}^{\circ}$ spécial, Zaïre: un pays à reconstruire, $41: 22-31$.

Retel-Laurentin (A.), 1974. Infécondité en Afrique Noire. Maladies et conséquences sociales. Masson, Paris, 188 p.

Romaniuk (A.), 1967. La fécondité des populations congolaises. Paris : Mouton, 348 p.

Sala Diakanda (M.), 1980. Approche ethnique des phénomènes démographiques : le cas du Zaïre. Recherches démographiques, Cahier n4, Cabay Librairie, Louvain la Neuve 433 p.

Tabutin (D.), 1982. Evolution régionale de de la fécondité dans l'Ouest du Zaïre. Population, vol. 1, 29-50.

TARDIEU (V.), 1992. Forêts des Hommes, Tropiques saccagés ou sauvés ? Robert Laffont, Paris 200216. 
Sala-Diakanda (M.), 1980. Approche ethnique des phénomènes démographiques; le cas du Zä̈re. Recherches démographiques, Cahier $n^{\circ} 4$, Cabay, Louvain-la-Neuve, 433 p.

SCHoEpf (B.G.), 1988. Women, AIDS and economic crisis in Central Africa. Canadian Journal of African Studies/Journal Canadien des Etudes Africaines, 22 : 625-644.

Schruvers (D.), Delaporte (E.), Peeters (M.), Dupont (A.), Meheus (A.), 1991. Seroprevalence of retroviral infection in women with different fertility statuses in Gabon, Western Eqyuatorial Africa. Journal of Acquired Immune Deficiency Syndromes, 4 : 468-470.

Songue (P.), 1986. Prostitution en Afrique, l'exemple de Yaoundé. Paris : L'Harmattan, Points de vue concrets, $154 \mathrm{p}$.

Verboud (M.), 1992. Les circuits de diffusion du virus en Afrique. Le Journal du Sida, pp. 16-17.

VINCKE (E.), 1991. Liquides sexuels féminins et rapports sociaux en Afrique Centrale. Anthropologie et Sociétés, 15: 167-188.

WERNER (J.F.), 1991. La prostitution en milieu urbain. Anthropologie et Sociétés, 15 : 255-262.

Reçu le 01/03/1997, accepté le 29/06/1998. 